Referat

\title{
Beeinflusst neonatal verabreichtes Morphin späteres Verhalten?
}

Ranger M, Synnes AR, Vinall j, Grunau RE. Internalizing behaviours in school-age children born very preterm are predicted by neonatal pain and morphine exposure. Eur J Pain 2014; 18: 844-852

Frühere Studien der Autorengruppe sollen nach eigenen Angaben zeigen, dass häufigere neonatale Schmerzerfahrungen bei Kindern, die vor der 33. Schwangerschaftswoche geboren wurden, in einem Alter von 18 Monaten zu vermehrten internalisierenden Verhaltensauffälligkeiten führen. Finden sich Verhaltensauffälligkeiten auch bei Schulkindern, die vor der 33. Schwangerschaftswoche zur Welt kamen?

Kanadische Pädiater untersuchten Erstklässler, die vor der 33. Woche geboren worden waren, und stellten auch bei den 6-8-Jährigen mehr internalisierendes Verhalten fest, je höher die postnatal eingesetzte Morphindosis war.

M. Ranger und Kollegen befragten dazu Eltern von 101 Kinder, die sie 6-8 Jahre zuvor auf ihrer Intensivstation als Frühgeborene behandelt hatten, mittels der CBCL (Children Behaviour Checklist; 113 Fragen). Zum internalisierenden Verhalten zählen Ängstlichkeit, Rückzugstendenzen, depressives Verhalten und somatische Probleme, während externalisiertes Verhalten sich etwa auf Aggressivität oder das Brechen von Regeln bezieht. In die Auswertung wurde nur internalisierendes Verhalten aufgenommen, da dieses als besonders von neonatalen Faktoren abhängig gilt. Zusätzlich beantworteten die Eltern den PSI-III (Parental Stress Index), von dem nur der elternbezogene Teil mit Themen wie Gesundheit, Familienverhältnisse, Rollenverteilung oder Depression mit in die Analyse einbezogen wurde.

Von den 101 Schulkindern

- hatten 57 neonatal eine künstliche Beatmung und Morphin erhalten.

- Die restlichen 44 Kinder hatten keine Beatmung benötigt und deshalb auch kein Morphin bekommen.

Die neonatale Belastung durch eventuell schmerzhafte Eingriffe wie Lanzettenpunktionen, aber auch das Legen einer Magensonde sowie die Morphindosis, wurden aus den Patientenakten retrospektiv entnommen.
- 77 der 101 Kinder zählten nach CBCL als unauffällig,

- 7 als grenzwertig und

- 17 als auffällig.

Dabei wurden zwischen den Gruppen der Kinder ohne und mit künstlicher Beatmung plus Morphineinsatz keine signifikanten Unterschiede gefunden, obschon die beatmeten Kinder 2-3-mal häufiger schmerzhaften Eingriffen ausgesetzt waren. Nach getrennter Analyse der Gruppen ergab sich jedoch

- bei den nicht beatmeten Kindern ein positiver und signifikanter Zusammenhang zwischen internalisierendem Verhalten im $\mathrm{CBCL}$, neonataler Schmerzbelastung und höherem elterlichen Stress und

- bei den beatmeten Kindern eine signifikante Beziehung zwischen höherem CBCL-Score für internalisierendes Verhalten und höherer verabreichter Morphinmenge.

Bei vorherigen Untersuchungen dieser Kinder im Alter von 18 Monaten hatten sich keine Assoziationen zwischen Höhe der Morphin-Expositionen und der Verhaltenseinschätzung durch die Eltern gefunden.

Abb. Kinderärzte in Kanada untersuchten 6- bis 8-jährige Kinder, die als Frühgeborene auf ihrer Intensivstation behandelt worden waren.

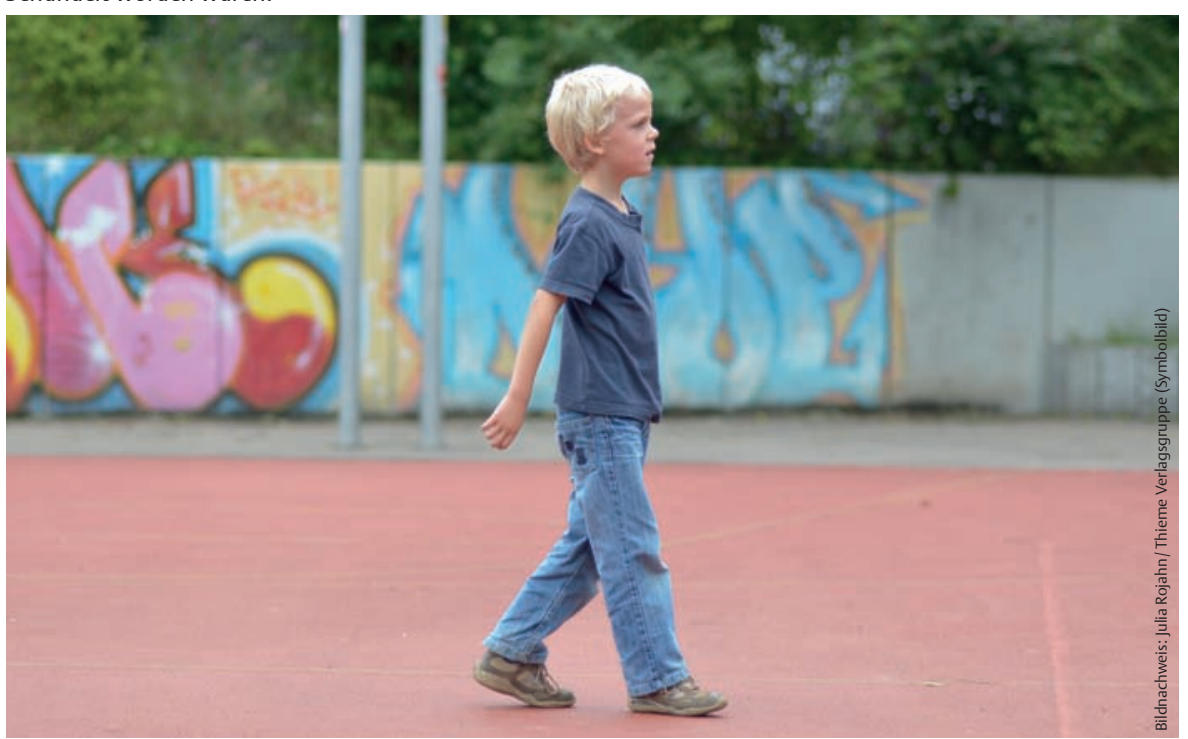

Fazit Die Studie weist eine Menge an Limitationen auf, die von den Autoren nicht berücksichtigt wurden; z. B. wurden die Daten nicht prospektiv erhoben, sondern retrospektiv. $15 \%$ der untersuchten Kinder zeigen einen auffälligen CBCL-Wert, was der Verteilung in der Normalbevölkerung entspricht. Zudem zeigten sich keine Unterschiede in der Anzahl relevant auffälliger CBCL-Werte zwischen der Gruppe von Kindern mit und ohne Morphin-Exposition bzw. mit sehr vielen und nur wenigen schmerzhaften Interventionen. Man würde sich prospektive multizentrische Studien wünschen, die den primären Endpunkt a priori definieren, eine Stichprobengröße kalkulieren, um Daten zu sammeln, die später mit einer zuvor festgelegten Methodik analysiert werden. Die Methodik der aktuellen Studie birgt das Risiko, dass die berichteten Ergebnisse auf Zufällen beruhen.

Dr. sc. hum. Katrin Wolf, Eitorf 


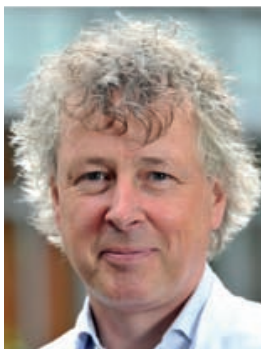

Prof. Dr. Boris Zernikow

Deutsches Kinder-

schmerzzentrum, Kinderschmerztherapie und Pädiatrische Palliativmedizin, Universität Witten/Herdecke
Beeinflusst die neonatale Opioidtherapie die spätere Entwicklung ehemaliger Frühgeborener? Viele Daten, wenige Patienten, keine Antworten!

Die Behandlung sehr kleiner Frühgeborener auf der Intensivstation ist eine extrem komplexe Intervention, die sich oft über Wochen hinzieht und geprägt ist von Instabilität und Unsicherheit. Beatmungsprobleme, Infektionen und andere Komplikationen wie nekrotisierende Enterokolitis, Hirnblutung oder Augenschäden bedrohen das Neugeborene und verursachen bei Eltern eine emotionale Achterbahnfahrt. Regelmäßig werden neue Entscheidungen getroffen, und Therapieanpassungen nötig. Eine unüberschaubare Menge an Patientendaten fällt an.

In der Studie von Ranger et al. wird versucht, die gesamte klinische Situation durch den Krankheitsschwere-Score SNAP II des ersten Lebenstages zu erfassen, um so die Analysen für diesen Faktor zu „korrigieren“. Das ist eine grobe und ungeeignete Simplifizierung. Indirekte Messinstrumente für die Krankheitsschwere oder Komplikationsbedrohung sind Anzahl und Ausmaß schmerzhafter Interventionen, die verbrauchte Opioidoder Katecholamindosis der ersten Lebenstage bis -wochen, mittlere Atemwegsdrücke etc. Sind solche Surrogatmarker, so wie in dieser und in anderen Studien, stark mit psychologischen Auffälligkeiten im späteren Leben korreliert, heißt das nur: Je mehr Opioide verbraucht wurden, desto kranker war das Kind und umso häufiger sind psychologische Auffälligkeiten im Kindergarten- oder Schulalter. Verwundert das wirklich? Mich nicht!
Zahlreiche Studien haben in der Vergangenheit versucht, verbrauchte Opioidgesamtdosis oder Anzahl schmerzhafter Interventionen als Einzelmaße mit dem neurologischen oder psychologischen Outcome der Frühgeborenen zu korrelieren. Das heißt, sie wollten zeigen, dass nicht die Krankheitsschwere, sondern allein die Schmerzerfahrung oder die Schmerztherapie korreliert sind mit diesem Outcome. Schon aus theoretischen und methodologischen Gründen muss ein solcher Versuch scheitern, weil die Zusammenhänge extrem komplex sind. Multivariate Untersuchungsmethoden - wie die multiple Regressionsanalyse - können eine solche Komplexität abbilden, benötigen jedoch für verlässliche Ergebnisse große Datenmengen. Es wird angenommen, dass mind. 50 Patienten pro untersuchtem Parameter notwendig sind, wenn die Ergebnisse mehr sein sollen als Zufallsfunde. In der vorliegenden Studie wurden 2 Gruppen mit n= 44 bzw. $n=57$ Kindern untersucht; gerade ausreichend für univariate Analysen, deren Signifikanzlevel dann noch nach Bonferroni korrigiert werden muss. Univariate Analysen in dieser Studie zeigten keine signifikanten Unterschiede zwischen Frühgeborenen, die Morphin erhalten hatten, und solchen ohne Morphintherapie. Das verwundert wirklich, weil die morphinbehandelten Kinder im Durchschnitt viel unreifer, leichter, am ersten Lebenstag kränker und dann beatmet waren und man erwarten darf, dass sie im späteren Leben mehr Gesundheitsprobleme und psychische Auffälligkeiten zeigen.
Der wichtigste Outcome-Parameter der Studie - die CBCL - ist ein Screeninginstrument, für das es einen klinisch relevanten Cut-offWert gibt. Die Häufigkeit der klinisch auffälligen Werte für internalisierendes Verhalten ist in beiden Gruppen gleich. In dieser Studie wurde die CBCL aber auch als ein kontinuierliches Maß verwendet. Über die inhaltliche Relevanz und Gültigkeit der CBCL als kontinuierliches Maß - d. h. der Einzelwerte unter und über dem Cut-off - gibt es keine ausreichenden Erkenntnisse. Die Annahme, dass die Ausprägung der Skala kontinuierlich ist, ist aber die Voraussetzung dafür, dass eine multiple Regressions- und nicht eine logistische Regressionsanalyse zum Einsatz kommt. Betrachtet man die Resultate des univariaten Gruppenvergleichs, hätte eine logistische Regression sicherlich kein „positives" Ergebnis gezeigt.

Abgesehen von diesen Limitationen der Studie ist natürlich die Empfehlung richtig, Morphindosen am Effekt und den aktuell zur Verfügung stehenden pharmakodynamischen und -kinetischen Daten auszurichten. Diese legen nahe, dass in der Neonatologie lange Zeit Morphin zu hoch dosiert wurde. Wichtig zum Verständnis der vorliegenden Arbeit ist zudem, dass Morphin in dieser Studie nicht primär zur Schmerztherapie, sondern hauptsächlich zur Sedierung während der Beatmung eingesetzt wurde (kanadische neonatal intensive care; NICU). Eine Indikation, die weltweit sehr umstritten und nicht evidenzbasiert ist. 